\title{
Entropic force and entanglement system
}

\author{
Yun Soo Myung 1 and Yong-Wan Kim² \\ Institute of Basic Science and School of Computer Aided Science, Inje University, \\ Gimhae 621-749, Republic of Korea
}

\begin{abstract}
We introduce the isothermal cavity, static holographic screen, and accelerating surface as holographic screen to study the entropic force in the presence of the Schwarzschild black hole. These may merge to provide a consistent holographic screen to define the entropic force on the stretched horizon near the event horizon. Considering the similarity between the stretched horizon of black hole and the entanglement system, we may define the entropic force in the entanglement system without referring the source mass.
\end{abstract}

\footnotetext{
${ }^{1}$ e-mail address: ysmyung@inje.ac.kr

2e-mail address: ywkim65@gmail.com
} 


\section{Introduction}

Since the discovery of the laws of black hole thermodynamics [1, Bekenstein [2] and Hawking [3] have suggested a deep connection between gravity and thermodynamics, realizing black hole entropy and Hawking radiation. Later on, Jacobson [4] has demonstrated that Einstein equations (describing relativistic gravitation) could be derived by combining general thermodynamic pictures with the equivalence principle. Padmanabhan [5] has observed that the equipartition law for horizon degrees of freedom combined with the Smarr formula leads to the Newton's law of gravity.

Recently, Verlinde has proposed the Newtonian force law as an entropic force (nonrelativistic version) by using the holographic principle and the equipartition rule [6]. If it is proven correct, gravity is not a fundamental interaction, but an emergent phenomenon which arises from the statistical behavior of microscopic degrees of freedom encoded on a holographic screen. In other words, the force of gravity is not something ingrained in matter itself, but it is an extra physical effect, emerging from the interplay of mass, time and space (information).

After his work, taking the apparent horizon as a holographic screen to derive the Friedmann equations [7], derivation of the Friedmann equations using the equipartition rule and unproved Unruh temperature [8, 9], the connection to the loop quantum gravity [10], the accelerating surfaces [11], holographic actions for black hole entropy [12], and application to holographic dark energy [13] were considered from the view of the entropic force. Furthermore, cosmological implications were reported in [14, 15, 16, 17, 18, 19], an extension to the Coulomb force [20], and the symmetry aspect of the entropy force [21] were investigated. The entropic force was discussed in the presence of black hole [22, 23, 24, 25].

However, one of urgent issues to resolve is to answer a question of how one can construct a spherical holographic screen of radius $R$ which encloses a source mass $M$ located at the origin to understand the entropic force. This is crucial because the holographic screen (an exotic description of spacetime) originates from relativistic approaches to black hole [26, 27] and cosmology [28]. Verlinde has introduced this screen by analogy with an absorbing process of a particle around the event horizon of black hole. Considering a smaller test mass $m$ located at $\Delta x$ away from the screen and getting the change of entropy on the screen, its behavior should resemble that of a particle approaching a stretched horizon of a black hole, as described by Bekenstein [2].

The next question is why the equipartition rule could be applied to this non-relativistic holographic screen to derive the Newtonian force law without any justifications. For black holes, the equipartition rule becomes the Smarr formula of $E=N T / 2=2 S T$ when using 
$N=4 S=A / G$ in the natural units of $\hbar=c=k_{B}=1$ and $G=l_{p l}^{2}$. Also it can be derived from the first law of thermodynamics $d E=T d S$ for the Schwarzschild black hole where the Komar charge is just the ADM mass $M$. Most of cosmological implications have used the holographic principle (screen) and equipartition rule to derive the Friedmann equations. However, these implications did not explain clearly how their approach is related to the appearance of the entropic force because these belong to the relativistic approach. Even though the equipartition rule is available for the Newtonian dynamics, the holographic principle of $N=A / G$ is not guaranteed to apply to any non-relativistic situations. In this sense, this issue is closely related to the first one.

If the above two questions are answered properly, one will make a further step to understand the entropic force. However, there is still a gap between non-relativistic and relativistic approaches. It seems that Verlinde was recycling some ideas for obtaining Einstein equations due to Jacobson's derivation of Einstein equations. Also, we would like to mention that he was using circular reasoning in his equations, by starting out with gravity.

At this time, it seems hard to discuss an entropic force without referring a holographic screen. Hence, we propose that introducing a holographic screen is a first step to understand the entropic force.

In this work, we show how a holographic screen is constructed to define the entropic force well by implementing the Schwarzschild spacetimes. Here, we introduce four candidates of the isothermal cavity, static holographic screen, accelerating surface, and stretched horizon to study the entropic force. Finally, we consider the entanglement system in the flat spacetime as a promising candidate for a holographic screen to define the entropic force without referring the mass $M$. We must say that our approach is not a complete scheme to understand the entropic force because we have already introduced a given configuration of gravitational field (relativistic situation), the Schwarzschild spacetimes to define a holographic screen.

We show that to define the entropic force, three of the isothermal cavity, static holographic screen, and accelerating surface might merge to provide a unified picture for a holographic screen on the stretched horizon near the event horizon. Considering a close relationship between black hole thermodynamics on the stretched horizon and the entanglement system, we propose that an entropic force may be defined in the entanglement system without referring the source mass $M$. 


\section{Entropic force}

In this section, we briefly review how the Newtonian force law emerges from entropic considerations. Explicitly, when a test particle with mass $m$ is located near a holographic screen with distance $\Delta x$, the change of entropy on a holographic screen may take the form

$$
\Delta S=2 \pi m \Delta x
$$

Considering that the entropy of a system depends on the distance $\Delta x$, an entropic force $F$ could be arisen from the thermodynamical conjugate of the distance as

$$
F \Delta x=T \Delta S
$$

which may be regarded as an indication that the first law of thermodynamics is realized on the holographic screen. Plugging (1) into (2) leads to an important connection between the entropic force and temperature

$$
F=2 \pi m T
$$

In this work, we use this connection mainly to derive the entropic force, after setting the temperature $T$ on the holographic screen.

Let us assume that the energy $E$ is distributed on a spherical screen with radius $R$ and the source mass $M$ is located at the origin of coordinates. Then, we assume that the equipartition rule [29, 5], the equality of energy and mass, and the holographic principle, respectively, hold as

$$
E=\frac{1}{2} N T, \quad E=M, \quad N=\frac{A}{G}=4 S
$$

with the area of a holographic screen $A=4 \pi R^{2}$. These are combined to provide the temperature on the screen

$$
T=\frac{G M}{2 \pi R^{2}} .
$$

Substituting (15) into (3) , one obtains the Newtonian force law as the entropic force

$$
F=\frac{G m M}{R^{2}}
$$

However, as was emphasized previously, the usage holographic screen is not guaranteed to describe a non-relativistic case of a source mass $M$. 


\section{Entropic force on the isothermal cavity}

In this section, to define a holographic screen, we consider the Schwarzschild spacetimes instead of the source mass $M$ as

$$
d s_{S c h}^{2}=g_{\mu \nu} d x^{\mu} d x^{\nu}=-\left(1-\frac{2 G M}{r}\right) d t^{2}+\frac{d r^{2}}{\left(1-\frac{2 G M}{r}\right)}+r^{2} d \Omega_{2}^{2} .
$$

Here the event horizon (EH) is located at $r=r_{E H}=2 G M$ whose horizon area is $A_{E H}=$ $4 \pi r_{E H}^{2}$.

It is well known that the Schwarzschild black hole could be in thermal equilibrium with a finite-size heat reservior in asymptotically flat spacetimes. This is made by introducing a cavity enclosed the black hole. In this case, we may introduce the local temperature (Tolman temperature) and the quasilocal energy on the isothermal cavity. Now let us introduce the Tolman redshift transformation on the black hole system [30]. Using this transformation, the local temperature observed by an observer located at $r>r_{E H}$ is defined by [31]

$$
T_{L}(r)=\frac{T_{\infty}}{\sqrt{-g_{t t}}}=\frac{1}{8 \pi G M} \frac{1}{\sqrt{1-\frac{2 G M}{r}}}
$$

where

$$
T_{\infty}=\frac{1}{8 \pi G M}=\frac{1}{4 \pi r_{E H}} \equiv T_{H}
$$

is the Hawking temperature $T_{H}$ measured by observer at infinity and the denominator of $\sqrt{-g_{t t}}$ is the redshift factor. It is worth to mention two limiting cases. On the cavity located at $r=r_{E H}+l_{p l}^{2} / r_{E H}$ near the event horizon, this local temperature is given approximately by $T=1 / 4 \pi l_{p l}$ which is independent of the black hole mass $M$ [32]. On the other hand, for $r \gg r_{E H}$, it reduces to the Hawking temperature $T_{H}$.

Similarly, the quasilocal energy is derived by considering the first law of thermodynamics and assuming that the Bekenstein-Hawking entropy $S_{B H}=4 \pi G M^{2}$ is not changed on the cavity

$$
d E_{Q L}(r)=T_{L}(r) d S_{B H},
$$

which is integrated to give

$$
E_{Q L}(r)=\frac{r}{G}\left[1-\sqrt{1-\frac{2 G M}{r}}\right] .
$$

Here the energy observed at infinity is surely the ADM mass $M$

$$
E_{Q L}(\infty)=M=\frac{r_{E H}}{2 G} .
$$


Solving $E_{Q L}$ for $M$ leads to

$$
M=E_{Q L}-\frac{G E_{Q L}^{2}}{2 r},
$$

which states that the ADM mass consists of the thermal energy and the gravitational self-energy to create a cavity at $r>r_{E H}$. In this sense, the isothermal cavity, which was an artificial device to make a phase transition from a hot gas to a black hole [30], is different from the holographic screen.

Consequently, it seems unlikely to define the entropic force on the isothermal cavity except the case that it located near the event horizon.

\section{Entropic force on the static holographic screen}

We briefly review how to derive the entropic force on the static holographic screen in the Schwarzschild spacetime (17). We introduce the proper acceleration [33]

$$
a^{\mu}=\xi^{\alpha} \xi_{; \alpha}^{\mu}
$$

where $\xi^{\alpha}$ is a timelike Killing vector field that satisfies $\xi_{\mu ; \nu}+\xi_{\nu ; \mu}=0$. We define an integral

$$
E(V)=\frac{1}{4 \pi} \oint_{\partial V} a^{\mu} n_{\mu} d A
$$

where $V$ is the bulk volume enclosed by spacelike hypersurface $\partial V=S^{2}$ and $n_{\mu}$ is a spacelike unit normal vector $S^{2}$. This can be rewritten as the Komar integral to define the concept of energy in the stationary spacetime

$$
E(V)=\frac{1}{8 \pi} \oint_{\partial V} \xi^{\mu ; \nu} d \Sigma_{\mu \nu}
$$

where

$$
d \Sigma_{\mu \nu}=\left(n_{\mu} \xi_{\nu}-n_{\nu} \xi_{\mu}\right) d A .
$$

For $r>r_{E H}$, a non-zero component of the Killing vector is given by

$$
\xi^{t}=1
$$

On the $S^{2}$, a non-zero component of the normal vector is

$$
n^{r}=-\sqrt{1-\frac{2 G M}{r}} .
$$


Using these, it is easily shown that the Komar integral (16) becomes the local energy which is defined on the holographic screen in the stationary spacetime [22]

$$
E_{H S}=\frac{E_{\infty}}{\sqrt{1-\frac{2 G M}{r}}}, \quad E_{\infty}=M .
$$

$E_{H S}$ indicates the energy of the gravitational field from the viewpoint of an observer at rest with respect to the Schwarzschild coordinate $r$. It is important to note that the local energy $E_{H S}$ defined on the holographic screen is not the quasilocal energy $E_{Q L}$ of (11) on the isothermal cavity. At this stage, we propose the local equipartition rule

$$
E_{H S}(r)=2 S_{H S}(r) T_{H S}(r)
$$

where the entropy $S_{H S}$ is defined on the holographic screen located at $r>r_{E H}$

$$
S_{H S}(r)=\frac{\pi r^{2}}{G}
$$

Then, the temperature on the holographic screen is given by

$$
T_{H S}(r)=\frac{G M}{2 \pi r^{2} \sqrt{1-\frac{2 G M}{r}}}
$$

which takes a different form from the local temperature $T_{L}(r)$ in Eq.(8). Plugging (23) into (3), we obtain the entropic force as

$$
F_{H S}=2 \pi m T_{H S}(r)=\frac{1}{\sqrt{1-\frac{r_{E H}}{r}}} \frac{G m M}{r^{2}}
$$

which shows that the mass $m$ feels an infinitely tidal force in the limit of $r \rightarrow r_{E H}$, while it takes the Newtonian force law at the large distance of $r \gg r_{E H}$. In this picture, it is hard to introduce a proper acceleration $a$ because one works in the stationary spacetime.

Consequently, in obtaining the entropic force (24), an important step was making use of the local equipartition rule (21) to assign the temperature on the static screen.

\section{Entropic force on the accelerating surface}

Now we are in a position to define the entropic force on the accelerating surface in the presence of the Schwarzschild black hole (77). In this case, the accelerating surface plays the role of the holographic screen. The accelerating surface was introduced in [33, 34]. Here 
we use the accelerating surface as the accelerating screen (AS). For the non-stationary spacetime, we introduce a future pointing unit vector

$$
u^{\mu}
$$

of the congruence for the timelike world lines of the points on $S^{2}$. It satisfies the orthogonality with the normal vector $n_{\mu}$ as

$$
u^{\mu} n_{\mu}=0
$$

Also this vector is necessary to define the change of the heat. In this case, a proper acceleration vector field is defined by

$$
a^{\mu}=u^{\alpha} u_{; \alpha}^{\mu}
$$

and an AS is defined by the following properties:

$$
\sqrt{a^{\mu} a_{\mu}}=a=\text { constant, } \quad a=a^{\mu} n_{\mu}
$$

at every points on $S^{2}$. The flux of the proper acceleration vector field through the AS is defined to be

$$
\Phi_{A S}=a A_{A S}
$$

where $A_{A S}$ is the area of the AS. A non-zero component of the future pointing unit vector is

$$
u^{t}=\frac{1}{\sqrt{1-\frac{2 G M}{r}}},
$$

and a non-zero component of the proper acceleration vector $a^{\mu}$ is given by

$$
a^{r}=u^{t} u_{; t}^{r}=-\frac{G M}{r^{2}} .
$$

The proper acceleration defined by

$$
a=a^{\mu} n_{\mu}=\frac{G M}{r^{2}} \frac{1}{\sqrt{1-\frac{2 G M}{r}}}
$$

plays the role of the Unruh temperature

$$
T_{U}(r)=\frac{a}{2 \pi}=\frac{G M}{2 \pi r^{2}} \frac{1}{\sqrt{1-\frac{2 G M}{r}}} .
$$

Considering the temperature (23) defined on the accelerating screen, we find that

$$
T_{H S}=T_{U}
$$


which means that the screen temperature $T_{H S}$ is equal to the bulk temperature $T_{U}$. A natural interpretation of this result is that an accelerating observer on the AS observes thermal radiation with the Unruh temperature $T_{U}$. This is a well-known result of the local quantum field theory, which is called the Unruh effect. This effect states that an accelerating observer always observes thermal particles even when, from the viewpoint of all inertial observers, there is no particles at all. In the Newtonian limit, it reduces to

$$
a=\frac{G M}{r^{2}}
$$

which is the acceleration of particles in freely falling in the gravitational field created by a point mass $M$. Then, the flux is given by

$$
\Phi_{A S}=\frac{4 G \pi M}{\sqrt{1-\frac{2 G M}{r}}}
$$

when using $A_{A S}=4 \pi r^{2}$.

Now let us introduce the change of the heat in terms of a differential $d \Phi_{A S}$ of the flux $\Phi_{A S}$ through the AS as

$$
\delta Q=\frac{1}{4 \pi G} d \Phi_{A S}
$$

Considering that the surface gravity $\kappa$ is constant on the event horizon, we keep $a$ fixed on the AS when varying the flux $\Phi_{A S}$. That is, $d a(r, M)=0$ implies

$$
d M=\frac{2 G M r-3(G M)^{2}}{r^{2}-G M r} \frac{d r}{G} .
$$

Using this, Eq.(37) leads to

$$
\delta Q=\frac{2 M}{r} \frac{1}{\sqrt{1-\frac{2 G M}{r}}} d r .
$$

In this case, the entropy is defined by

$$
S_{A S}=\frac{A}{2 G}=\frac{2 \pi r^{2}}{G}
$$

and thus, its differential is $d S_{A S}=4 \pi r d r / G$. Plugging this into (39) leads to the fact that the change of the heat is balanced by the change of the entropy when fixing the temperature on the AS

$$
\delta Q=\frac{G M}{2 \pi r^{2}} \frac{1}{\sqrt{1-\frac{2 G M}{r}}} d S_{A S}=T_{U}(r) d S_{A S}=\frac{a}{2 \pi} d S_{A S} .
$$

We confirm that the entropic force on the point mass $m$ near the AS is given by

$$
F_{H S}=2 \pi m T_{U}=m a=\frac{1}{\sqrt{1-\frac{2 G M}{r}}} \frac{G m M}{r^{2}} .
$$




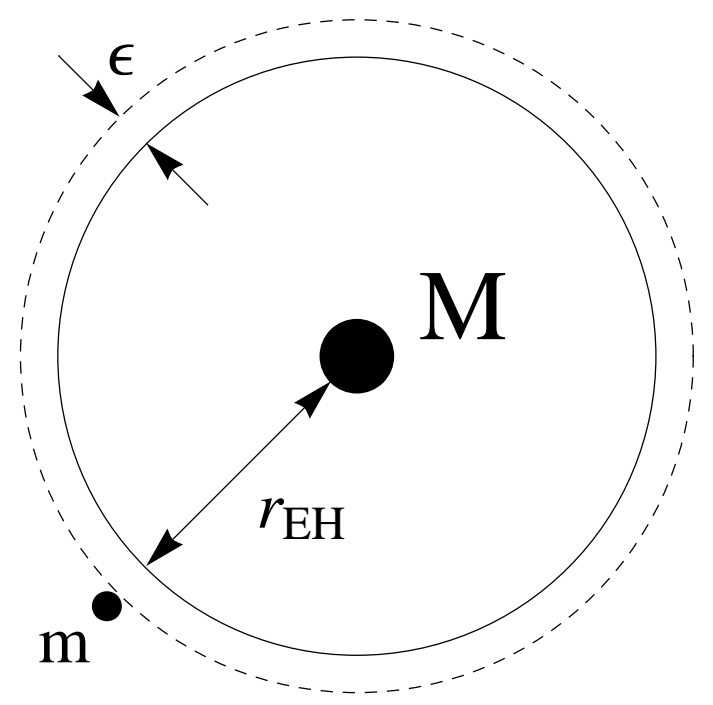

Figure 1: The Schwarzschild black hole is present by noting the event horizon $r_{E H}$ (solid circle). At the position $r=r_{E H}+l_{p l}^{2} / r_{E H}$ near the event horizon, equality of isothermal cavity=static holographic screen=accelerating screen is depicted in terms of the dotted circle in order to define the entropic force.

Finally, we observe that if one introduces the accelerating surface as the accelerating screen, it is very natural to define the acceleration $a$ of a test particle $m$ through the Unruh temperature $T_{U}$ and thus, the entropic force (24) is recovered properly.

\section{Entropic force on the stretched horizon}

Since the isothermal cavity, static holographic screen, and the accelerating screen do not provide a consistent form like the entropic force (6) , it is natural to ask where is the specific place to give a consistent form. In this section, we suggest that this place is just on the stretched horizon ( $\mathrm{SH}$ ) [35]. This means that all thermodynamic quantities are measured by an observer located at the proper distance $l_{p l}$ away from the horizon.

As is shown in Fig. 1, this horizon is considered as the holographic screen at $r=$ $r_{E H}+l_{p l}^{2} / r_{E H}$ near the event horizon. The length contraction of $l_{p l}^{2} / r_{E H}=\sqrt{-g_{t t}} l_{p l}$ is due to the redshift transformation of $\sqrt{-g_{t t}} \simeq l_{p l} / r_{E H}$ near the horizon. On the SH, the 
local temperature is given by

$$
T_{L}^{S H}=\frac{1}{4 \pi l_{p l}} \sqrt{1+\frac{l_{p l}^{2}}{r_{E H}^{2}}} \simeq \frac{1}{4 \pi l_{p l}}\left(1+\frac{1}{2} \frac{l_{p l}^{2}}{r_{E H}^{2}}\right)=\frac{1}{4 \pi l_{p l}}\left(1+\frac{1}{8 l_{p l}^{2} M^{2}}\right)
$$

which is independent of the black hole mass $M$ in the leading order [32]. On the other hand, the HS temperature (=Unruh temperature) leads to

$$
T_{H S}^{S H}=T_{U}^{S H}=\frac{1}{4 \pi l_{p l}\left(1+\frac{l_{p l}^{2}}{r_{E H}^{2}}\right)^{\frac{3}{2}}} \simeq \frac{1}{4 \pi l_{p l}}\left(1-\frac{3}{2} \frac{l_{p l}^{2}}{r_{E H}^{2}}\right) .
$$

Importantly, we observe that three temperatures are the same

$$
T_{L}^{S H}=T_{H S}^{S H}=T_{U}^{S H}=\frac{1}{4 \pi l_{p l}}
$$

in the leading order. Similarly, the local energy is given by

$$
E_{H S}^{S H}=\frac{r_{E H}^{2}}{2 l_{p l}^{3}} \sqrt{1+\frac{l_{p l}^{2}}{r_{E H}^{2}}} \simeq \frac{A_{E H}}{8 \pi l_{p l}^{3}}\left(1+\frac{1}{2} \frac{l_{p l}^{2}}{r_{E H}^{2}}\right)
$$

In the leading order, we check easily that the equipartition rule is satisfied as is shown by

$$
E_{H S}^{S H}=\frac{A_{E H}}{8 \pi l_{p l}^{3}}=\frac{N^{S H} T_{H S}^{S H}}{2}
$$

with $N^{S H}=A_{E H} / l_{p l}^{2}$. However, this shows a different case when comparing with (44) on the holographic screen for a non-relativistic case. Especially, the energy and temperature take different forms

$$
E_{H S}^{S H}=2 l_{p l} M^{2}, \quad T_{H S}^{S H}=\frac{1}{4 \pi l_{p l}}
$$

which show that the energy is proportional to mass squared $M^{2}$, while the temperature is independent of the mass. We remind the reader that this is a feature of thermodynamic quantities of black hole on the stretched horizon, which can be approximated by the Rindler spacetimes where a commutation relation of $[M, t]=i$ is defined for the Schwarzschild mass and time. Here, $E_{H S}^{S H}$ is the Rindler energy as defined by an observer near the horizon, while $M$ is the Schwarzschild mass (energy) as measured by an observer at infinity. Importantly, we observe that the first law of thermodynamics holds on the stretched horizon as

$$
d E_{H S}^{S H}=T_{H S}^{S H} d S^{S H}
$$


Here the entropy on the stretched horizon is given by

$$
S^{S H}=\frac{A_{E H}}{2 l_{p l}^{2}},
$$

which is clearly different from the the Bekenstein-Hawking entropy

$$
S_{B H}=\frac{A_{E H}}{4 l_{p l}^{2}} .
$$

Finally, the entropic force is consistently defined by

$$
F_{S H}=2 \pi m T_{L}^{S H}=2 \pi m T_{H S}^{S H}=2 \pi m T_{U}^{S H}=\frac{m}{2 l_{p l}},
$$

which takes a familiar form of

$$
F_{S H}=m a_{S H}
$$

with the proper acceleration $a_{S H}=\frac{1}{2 l_{p l}}$ on the stretched horizon.

However, we note that the quasilocal energy takes a different form on the SH

$$
E_{Q L}^{S H} \simeq \frac{1}{2 l_{p l} M}\left(1-\frac{1}{2} \frac{l_{p l}^{2}}{r_{E H}^{2}}\right)
$$

when comparing with the local energy in Eq.(48). The equipartition rule is not satisfied, as is evident from $E_{Q L}^{S H} \neq 2 S_{B H} T_{L}^{S H}$ in the leading order. Therefore, it seems that the quasilocal energy is not directly related to the entropic force.

\section{Entanglement system}

Entanglement entropy is a general concept which is a coarse graining entropy for a quantum system caused by an observer's partial ignorance of the information on state [36]. For an entanglement system in the flat spacetime, we consider the three-dimensional spherical volume $V$ and its enclosed boundary $\mathcal{B}=S^{2}$ (See Fig. 2). We assume that this system with radius $R$ and the cutoff scale $b$ is described by the local quantum field theory of a free scalar field $\phi$. The entanglement energy is carried by the modes around $\mathcal{B}$, which implies that the cutoff scale $b$ is introduced only in the $r$ direction through the length contraction $b^{2} / R^{2}$. We start by noting the similarity between the entanglement system in the flat spacetime and the stretched horizon formulation of the Schwarzschild black hole. Here we introduce the first law of thermodynamics for the entanglement system

$$
d E_{E N T}=T_{E N T} d S_{E N T}
$$




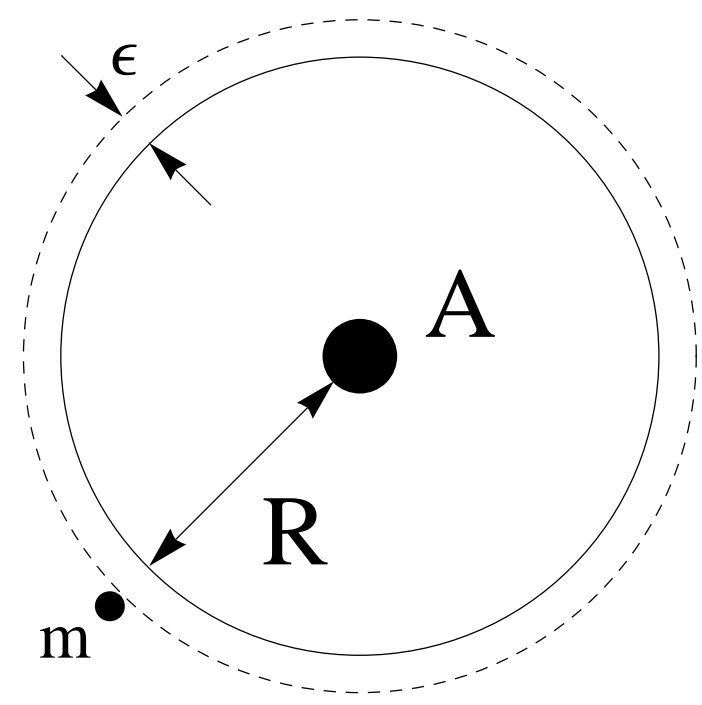

Figure 2: The entanglement system is present by noting the boundary $\mathcal{B}$ of area $A=4 \pi R^{2}$ (solid circle) enclosed the volume $V$. At the position $r=R+b^{2} / R$ near the boundary, a screen is depicted in terms of the dotted circle to define the entropic force.

whose thermodynamic quantities are given by [37]

$$
S_{E N T} \simeq \frac{A}{b^{2}}, E_{E N T} \simeq \frac{A}{b^{3}}, T_{E N T} \simeq \frac{1}{b},
$$

where $A=4 \pi R^{2}$ is the area of the boundary $\mathcal{B}$ of the system. Eq.(56) shows a universal behavior for all entanglement systems in Minkowski spacetime. We note that the zeropoint energy of the system was subtracted in the calculation of the entanglement energy $E_{E N T}$, thus degrees of freedom on the boundary contribute to giving $E_{E N T} \sim A$. It seems that its areal behavior is compatible with the concept of the entanglement. The entanglement entropy behaves universally as $S_{E N T} \sim A$, which takes the same form as that of the black hole. This is why the entanglement entropy is considered as the origin of the black hole entropy. The entanglement temperature is independent of the radius $R$ of system. Considering the connection between $R \leftrightarrow r_{E H}$ and $b \leftrightarrow l_{p l}$, thermodynamic quantities are the nearly same as those of the black hole on the stretched horizon:

$$
S_{E N T} \leftrightarrow S^{S H}, \quad E_{E N T} \leftrightarrow E_{H S}^{S H}, \quad T_{E N T} \leftrightarrow T_{H S}^{S H}=T_{L}^{S H}=T_{U}^{S H} .
$$

In order to compare (56) with those for the black hole observed at infinity, one observes that entanglement quantities at $r=R+b^{2} / R$ are blueshifted with respect to 
those at infinity by inserting the factor of $1 / \sqrt{-g_{t t}}$. With $\sqrt{-g_{t t}} \simeq b / R$, one obtains $E_{E N T}^{\infty} \simeq \sqrt{-g_{t t}} E_{E N T}=R / b^{2}$ and $T_{E N T}^{\infty} \simeq \sqrt{-g_{t t}} T_{E N T}=1 / R$ [32, 39]. It shows how an inclusion of gravity alters thermodynamics of the entanglement system. Later on, the same authors have calculated the entanglement energy in the Schwarzschild background without introducing the redshift factor [38].

Comparing the entanglement system with the stretched horizon of the Schwarzschild black hole, two are very similar to each other even though the entanglement system does not have the source mass $M$ and thus, it has no effects of gravity definitely. Importantly, we may consider the entanglement system as a genuine system to define the entropic force. This is because we could define the entropic force in the entanglement system without introducing the mass $M$. In this case, the equipartition rule

$$
E_{E N T} \simeq N_{E N T} T_{E N T}=\frac{A}{b^{2}} \frac{1}{b}
$$

is very special because the energy is proportional to the area $A$ (but not the mass $M$ ) and the temperature is independent of the area.

Finally, we propose that an entropic force could be defined by

$$
F_{E N T}=2 \pi m T_{E N T} \simeq \frac{m}{2 b},
$$

which takes another form of

$$
F_{E N T} \simeq m a_{E N T}
$$

with the proper acceleration $a_{E N T}=\frac{1}{2 b}$ in the flat spacetime. This proposition may be a good idea to ensure the entropic force if the latter does really exist.

\section{Discussions}

It is fare to say that the origin of the gravity is not yet fully understood. If the gravity is not a fundamental force, it may be emergent from the other approach to gravity. Newtonian force law could be emergent from the equipartition rule and the holographic principle [6]. However, one important issue is whether the holographic screen could be nicely defined for a non-relativistic case of a source mass $M$.

In this work, in order to realize the entropic force, we have first introduced the isothermal cavity, static holographic screen, and accelerating screen as a candidate for the holographic screen by implementing the Schwarzschild spacetimes. Then, using Eq.(3), we have found the entropic force (24) and (42) which are different from the original form (6). 
Furthermore, these might merge to provide a unified picture to define the entropic force (52) on the stretched horizon near the event horizon.

It is well-known that the entanglement system also respects the equipartition rule and the holographic principle in the flat spacetime [37]. Considering a close relationship between black hole thermodynamics on the stretched horizon and the entanglement system, we have proposed that an entropic force could be realized as (59) in the entanglement system without referring a source mass $M$.

Finally, we should say that our approach is not a complete scheme to understand the entropic force because we have already introduced a given configuration of gravitational field (Schwarzschild spacetimes), to define the holographic screen. However, given the holographic screen properly as was in this work, one could define the entropic force by using (3).

\section{Acknowledgment}

The author thanks Rong-Gen Cai for helpful discussions on an entropic force. This work was supported by Basic Science Research Program through the National Research Foundation (NRF) of Korea funded by the Ministry of Education, Science and Technology (2009-0086861).

\section{References}

[1] J. M. Bardeen, B. Carter and S. W. Hawking, Commun. Math. Phys. 31, 161 (1973).

[2] J. D. Bekenstein, Phys. Rev. D 7, 2333 (1973).

[3] S. W. Hawking, Commun. Math. Phys. 43, 199 (1975) [Erratum-ibid. 46, 206 (1976)].

[4] T. Jacobson, Phys. Rev. Lett. 75, 1260 (1995) arXiv:gr-qc/9504004].

[5] T. Padmanabhan, arXiv:0912.3165 [gr-qc].

[6] E. P. Verlinde, arXiv:1001.0785 [hep-th].

[7] F. W. Shu and Y. Gong, arXiv:1001.3237 [gr-qc].

[8] T. Padmanabhan, arXiv:1001.3380 [gr-qc]. 
[9] R. G. Cai, L. M. Cao and N. Ohta, Phys. Rev. D 81, 061501(R) (2010) arXiv:1001.3470 [hep-th]].

[10] L. Smolin, arXiv:1001.3668 [gr-qc].

[11] J. Makea, arXiv:1001.3808 [gr-qc].

[12] F. Caravelli and L. Modesto, arXiv:1001.4364 [gr-qc].

[13] M. Li and Y. Wang, Phys. Lett. B 687, 243 (2010) arXiv:1001.4466 [hep-th]].

[14] C. Gao, arXiv:1001.4585 [hep-th].

[15] Y. Zhang, Y. g. Gong and Z. H. Zhu, arXiv:1001.4677 [hep-th].

[16] Y. Wang, arXiv:1001.4786 [hep-th].

[17] S. W. Wei, Y. X. Liu and Y. Q. Wang, arXiv:1001.5238 [hep-th].

[18] Y. Ling and J. P. Wu, arXiv:1001.5324 [hep-th].

[19] J. W. Lee, H. C. Kim and J. Lee, arXiv:1001.5445 [hep-th].

[20] T. Wang, arXiv:1001.4965 [hep-th].

[21] L. Zhao, arXiv:1002.0488 [hep-th].

[22] Y. S. Myung, arXiv:1002.0871 [hep-th].

[23] Y. X. Liu, Y. Q. Wang and S. W. Wei, arXiv:1002.1062 [hep-th].

[24] Y. Tian and X. Wu, arXiv:1002.1275 [hep-th].

[25] R. G. Cai, L. M. Cao and N. Ohta, Phys. Rev. D 81, 084012 (2010) arXiv:1002.1136 [hep-th]].

[26] G. 't Hooft, arXiv:gr-qc/9310026.

[27] L. Susskind, J. Math. Phys. 36, 6377 (1995) arXiv:hep-th/9409089].

[28] R. Bousso, Rev. Mod. Phys. 74, 825 (2002) arXiv:hep-th/0203101.

[29] T. Padmanabhan, Class. Quant. Grav. 21, 4485(2004) arXiv:gr-qc/0308070.

[30] G. W. Gibbons, M. J. Perry and C. N. Pope, Phys. Rev. D 72, 084028 (2005) hep-th/0506233. 
[31] J. W. York, Phys. Rev. D 33,2092 (1986).

[32] Y. S. Myung, Phys. Lett. B 636, 324 (2006) arXiv:gr-qc/0511104.

[33] J. Makela, arXiv:0805.3955 [gr-qc].

[34] J. Makela, arXiv:0810.4910 [Unknown].

[35] L. Susskind and J. Lindesay, Black holes, information, and the string theory revolution (World Scientific, Singapore, 2005).

[36] L. Bombelli, R. K. Koul, J. H. Lee and R. D. Sorkin, Phys. Rev. D 34, 373 (1986).

[37] S. Mukohyama, M. Seriu and H. Kodama, Phys. Rev. D 55, 7666 (1997) gr-qc/9701059].

[38] S. Mukohyama, M. Seriu and H. Kodama, Phys. Rev. D 58, 064001 (1998) gr-qc/9712018.

[39] Y. S. Myung and M. G. Seo, Phys. Lett. B 671, 435 (2009) [arXiv:0803.2913 [gr-qc]]. 